\title{
An engineered cyclic peptide alleviates symptoms of inflammation in a murine model of inflammatory bowel disease
}

Received for publication, February 2, 2017, and in revised form, April 20, 2017 Published, Papers in Press, May 4, 2017, DOI 10.1074/jbc.M117.779215

Claudia Cobos Caceres, Paramjit S. Bansal, Severine Navarro, David Wilson, Laurianne Don, Paul Giacomin, Alex Loukas' ${ }^{1}$, and Norelle L. Daly ${ }^{2}$

From the Centre for Biodiscovery and Molecular Development of Therapeutics, AlTHM, James Cook University, Cairns, Queensland 4870, Australia

Edited by Wolfgang Peti

Inflammatory bowel diseases (IBDs) are a set of complex and debilitating diseases for which there is no satisfactory treatment. Recent studies have shown that small peptides show promise for reducing inflammation in models of IBD. However, these small peptides are likely to be unstable and rapidly cleared from the circulation, and therefore, if not modified for better stability, represent non-viable drug leads. We hypothesized that improving the stability of these peptides by grafting them into a stable cyclic peptide scaffold may enhance their therapeutic potential. Using this approach, we have designed a novel cyclic peptide that comprises a small bioactive peptide from the annexin A1 protein grafted into a sunflower trypsin inhibitor cyclic scaffold. We used native chemical ligation to synthesize the grafted cyclic peptide. This engineered cyclic peptide maintained the overall fold of the naturally occurring cyclic peptide, was more effective at reducing inflammation in a mouse model of acute colitis than the bioactive peptide alone, and showed enhanced stability in human serum. Our findings suggest that the use of cyclic peptides as structural backbones offers a promising approach for the treatment of IBD and potentially other chronic inflammatory conditions.

Inflammatory bowel diseases (IBDs) ${ }^{3}$ are a set of chronic inflammatory disorders of the gastrointestinal tract; the two major forms are ulcerative colitis and Crohn's disease (1). Although there are several medications on the market for IBD, they generally only provide temporary relief, and 70\% of IBD patients require surgical intervention (2). Because the current

The authors declare that they have no conflicts of interest with the contents of this article.

This article contains supplemental Tables 1 and 2 .

The atomic coordinates and structure factors (codes 5VAV and 5VFW) have been deposited in the Protein Data Bank (http://wwpdb.org/).

The chemical shifts are available in the Biological Magnetic Resonance Data Bank under accession numbers 30274 and 30281.

${ }^{1}$ Recipient of fellowship support from National Health and Medical Research Council NHMRC (1020114). To whom correspondence should be addressed. Tel.: 61-7-4232-1608; Email: alex.loukas@jcu.edu.au.

${ }^{2}$ Recipient of fellowship support from Australian Research Council (FF110100226). To whom correspondence should be addressed. Tel.: 61-742321815; E-mail: norelle.daly@jcu.edu.au.

${ }^{3}$ The abbreviations used are: IBD, inflammatory bowel disease; SFTI-1, sunflower trypsin inhibitor-1; DMF, dimethylformamide; TNBS, 2,4,6-trinitrobenzenesulfonic acid; Fmoc, fluorenylmethyloxycarbonyl; TOCSY, total correlation spectroscopy; RP-HPLC, reverse-phase HPLC. treatments are not satisfactory, new drug leads are being sought from a range of sources, including small molecules from plants and bioactive regions of larger proteins (3-6).

Intriguingly, several small peptides, some comprising only three residues, have been shown to be effective in the treatment of experimental colitis in mice (7-11). One example is a tripeptide (MC-12) derived from annexin A1, a calcium-dependent phospholipid-binding, anti-inflammatory protein (12). Annexin A1 mediates expression of cytokines such as TNF- $\alpha$, IL-6, and IL-10. It is required for the inhibition of NF- $\kappa$ B activity by anti-inflammatory drugs (13) and has recently been shown to be a potential biomarker of therapeutic efficacy for IBD (14). The $\mathrm{N}$-terminal region of annexin A1 comprising residues $2-26$ (Ac2-26) has been well studied $(15,16)$ and appears to have the same biological effects as the full-length protein (17). Analysis of a series of peptides based on the N-terminal sequence of annexin A1 showed that MC-12 (Ac-Gln-Ala-Trp) was the most potent inhibitor of NF- $\kappa$ B activity in SW480 cells (13).

Small peptides such as MC-12 are likely to be unstable and not viable drug leads, supported by the finding that high doses $(25 \mathrm{mg} / \mathrm{kg})$ of MC-12 were required to elicit an in vivo response in mouse colitis models, and it was more effective when injected compared with oral administration (18). Improving the stability of MC-12 and related peptides may enhance their therapeutic potential.

A range of approaches has been used to improve the stability of peptides, including backbone cyclization, and grafting into cyclic peptide scaffolds. Both approaches are based on the inherent stability observed for naturally occurring cyclic peptides such as cyclosporine, an 11-residue cyclic peptide, used clinically as an immunosuppressant agent. Sunflower trypsin inhibitor 1 (SFTI-1) is an example of a cyclic peptide that has proven useful in grafting studies. SFTI-1 was originally isolated from the seeds of sunflowers (Helianthus annuus), contains 14 residues and is one of the most potent trypsin inhibitors known (19). The structure comprises two short antiparallel $\beta$-strands that are linked by a single disulfide bond. The two loops formed by the cyclic backbone and disulfide bond are termed the "binding" loop, which contains the active-site lysine residue for binding to trypsin, and the "cyclization" loop, which contains the residues involved in the backbone cyclization. In addition to the cyclic backbone and disulfide bond, SFTI-1 contains a network 


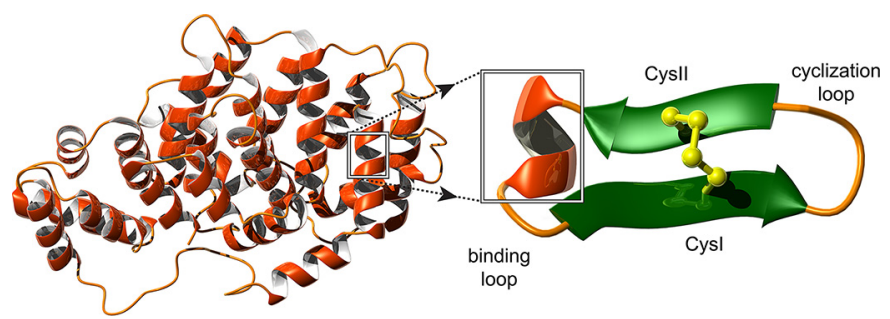

Figure 1. Schematic representation of grafting into the SFTI-1 scaffold. The three-dimensional structure of annexin $\mathrm{A} 1$ is shown on the left of the diagram (PDB ID code 1HM6). MC-12, highlighted on the structure of annexin A1, forms a helical structure in the full-length protein. The helical region of MC-12 is schematically represented as grafted into the binding loop of SFTI-1. SFTI- 1 comprises two $\beta$-strands connected by a disulfide bond. The cyclization loop is also labeled on the diagram. The figure was generated using MOLMOL (37).

of hydrogen bonds, all of which make the peptide highly stable (19). Amino acid substitutions are structurally well tolerated, and the protease inhibitory activity can be removed by mutation of the active-site lysine residue from the binding loop (20). The stability and tolerance to sequence changes makes SFTI-1 a promising peptide drug scaffold (19, 21, 22). Engineered forms of SFTI-1 have been developed as inhibitors of the serine proteases KLK4, KLK5, and KLK7, which are possible targets for cancer treatment $(21,23)$ as well as in the development of angiogenic agents (22), anti-angiogenic agents (24), and matriptase inhibitors (25).

Here we show that grafting the tripeptide MC-12 into the SFTI-1 scaffold improves its therapeutic efficacy in a murine model of chemically induced acute colitis while also improving its in vitro stability. A schematic representation of the grafting approach is shown in Fig. 1, highlighting the helical structure of MC-12 in annexin A1. A range of peptides was synthesized to explore the importance of the cyclic backbone and the loop into which the sequence was grafted.

\section{Results}

\section{Peptide design and synthesis}

The tripeptide MC-12 was grafted into the SFTI-1 cyclic scaffold with the aim of improving its stability and potency. MC-12 was grafted into the binding loop of SFTI-1, as this resulted in removal of the $\mathrm{P} 1$ lysine residue. Acyclic versions of SFTI-1 incorporating the MC-12 sequence were also designed to examine the influence of the cyclic backbone and loopgrafted on structure and activity. Ac2-26 was also synthesized using Fmoc chemistry to provide additional insight into the structure function relationships of the MC-12 sequence. The sequences of the synthetic peptides are shown in Fig. 2.

\section{Structural analysis using NMR spectroscopy}

The structures of all the peptides were analyzed using NMR spectroscopy. In general, the peptides displayed significant dispersion in the amide region and a single set of resonances for each residue, consistent with peptides containing $\beta$-sheet structure and one well defined conformation. By contrast, linMC12(p) had significant overlap in the amide region, which prevented assignment of the resonances. With the exception of lin-MC12(p), two-dimensional spectra (TOCSY and NOESY) allowed assignment of the resonances, and the secondary

\author{
SFTI-1 \\ cyc-MC12 \\ $\operatorname{lin}-\mathrm{MC12}(\mathrm{n})$ \\ $\operatorname{lin}-\mathrm{MC12}(\mathrm{p})$
}

lin-MC12(I2)

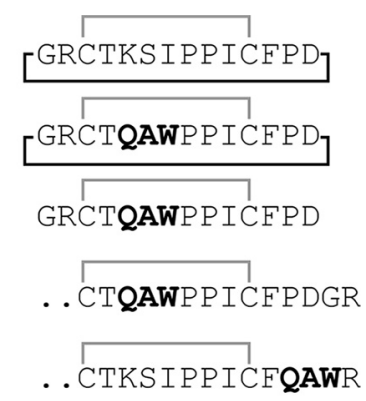

Figure 2. Sequences of the grafted peptides. The sequences of SFTI-1 (19), and the grafted peptides are shown. The MC- 12 sequence is highlighted in bold. The disulfide bond linking the two cysteine residues is shown in gray, and the cyclic backbone is shown with a black line.

chemical shifts (secondary shifts) were determined by subtracting random coil chemical shifts (26) from the $\alpha \mathrm{H}$ chemical shifts. A comparison of the secondary shifts is shown in Fig. 3. The secondary shifts were similar between cyc-MC12 and linMC12(n), but lin-MC12(12) had significantly different shifts for the cysteine residues compared with the other two peptides. The cysteine residues form part of the $\beta$-hairpin in the native peptide and consequently had downfield-shifted $\alpha$ protons. The lack of downfield-shifted $\alpha$ protons for the cysteine residues in lin-MC12(12) suggests that the overall fold differs from the native peptide.

Despite the similarities in secondary shifts between cycMC12 and lin-MC12(n), analysis of the amide exchange rates shows a significant difference as shown in Table 1 . The exchange rates for lin-MC12(n) were higher than those observed for cyc-MC12, indicating that the overall structural stability had been decreased.

The three-dimensional structures of cyc-MC12 and Ac2-26 were determined using the program CYANA based on distance restraints from the NOESY spectrum and angle restraints derived from TALOS + . The 20 lowest energy structures were chosen to represent the structures of cyc-MC12 and Ac2-26. Cyc-MC12 had a well defined structure, including the loop containing the grafted residues as shown in Fig. 4. The structure overlapped with the backbone atoms of SFTI-1 with a root mean square deviation of $1.32 \AA$, highlighting the similarity between the two peptides. The structure statistics are provided as supplemental Table 1. The structures of Ac2-26 are most well defined over the first 13 residues with a root mean square deviation of 0.08 Å over the backbone atoms as shown in Fig. 5. An $\alpha$-helix is present from residues 7-10, with residues 9 and 10 corresponding to the Gln and Ala of MC-12. A $3_{10}$ helix is also present between residues $15-17$. The structure statistics are provided as supplemental Table 2.

\section{2,4,6-Trinitrobenzenesulfonic acid (TNBS) mouse model}

The TNBS-induced murine colitis model was used to test the biological activities of all peptides. Mice were either left untreated (naïve) or were treated with peptide $5 \mathrm{~h}$ before administration of TNBS. On day 3 of the experiment, all mice were euthanized by gas asphyxiation and examined. Mice treated with TNBS alone lost weight within 1 day of TNBS administration and continued to lose weight until day three. Initial experiments used $1 \mathrm{mg} / \mathrm{kg}$ body weight dosage for cyc- 




Figure 3. Secondary shift analysis of peptides. The secondary shifts for SFTI-1 are shown in red, cyc-MC12 in blue, lin-MC12(n) in cyan, and linMC12(12) in yellow. The secondary shifts were calculated by subtracting the random coil shifts (26) from the $\alpha \mathrm{H}$ shift. The overall shifts are similar for all peptides with the exception of lin-MC12(I2), where the shifts for the cysteine residues (residues 3 and 11) differ significantly from the other peptides.

\section{Table 1}

Experimental amide exchange rates $\left(k_{\text {ex }} \min ^{-1}\right)$ for cyc-MC12 and lin-MC12 (n)

\begin{tabular}{lll}
\hline Residue & Cyc-MC12 & Lin-MC12(n) \\
Gly-1 & \multicolumn{1}{c}{$\times 10^{-3}$} & $\times 10^{-3}$ \\
Cys-3 & 0.56 & \\
Thr-4 & 1.2 & 61 \\
Ala-6 & 1.8 & \\
Trp-7 & & 13 \\
Ile-10 & 0.37 & 1.2 \\
Cys-11 & 3.5 & 69 \\
Phe-12 & 0.53 & 33 \\
\hline
\end{tabular}

MC12, but only partial protection in the TNBS model was observed (results not shown), so subsequent experiments used $3 \mathrm{mg} / \mathrm{kg}$. Mice that were treated with $60 \mu \mathrm{g}(3 \mathrm{mg} / \mathrm{kg})$ of cycMC12 before administration of TNBS displayed significantly reduced weight loss at day 3 , whereas all other peptides tested, including the linear form of the $\mathrm{MC}-12$ peptide, had no significant effect (Fig. 6A). Consistent with enhanced protection against TNBS-induced colitis, cyc-MC12-treated mice had visibly longer, healthier looking colons than untreated control mice administered TNBS (Fig. 6B). The length of the colon in cyc-MC12-treated mice was similar to that of naïve mice (Fig. $6 C$ ). Colons were then scored macroscopically by the following parameters: adhesions, bowel wall thickening, mucosal edema, ulceration, necrosis, and colon length (macroscopic score); they demonstrated that cyc-MC12-treated mice displayed a significantly reduced macroscopic score (Fig. 6D). SFTI-1 displayed no protective effect, indicating that the effect observed as a result of administering cyc-MC12 was related to the grafted region rather than the native scaffold. The tripeptide MC-12 administered at a dose of $60 \mu \mathrm{g}$ did not have an influence on weight loss or macroscopic score. However, the colon length of MC-12-treated mice was significantly longer than the TNBS-only treated mice, albeit not to the same extent as the cyc-MC12 peptide. The colon lengths and the macroscopic pathology scores of mice treated with Ac2-26 showed a protective effect but not to the same extent as cyc-MC12.

\section{Serum stability}

The stability of the peptides in human serum was assessed over an 8-h period as shown in Fig. 7. MC-12 and annexin A1(2-26) were completely degraded after $8 \mathrm{~h}$. By contrast, cycMC12 was stable in human serum over the time course of the experiment. The acyclic peptides were more stable than MC-12 and Ac2-26, but were degraded to $\sim 60 \%$ of the initial concentration within the first $3 \mathrm{~h}$ of incubation. The lower stability of the linear peptides demonstrates that the disulfide bond alone is not sufficient to confer high stability and that the cyclic backbone enhances the stability of the grafted peptides in human serum.

\section{Discussion}

This study broadens the knowledge on grafting bioactive sequences into stable cyclic peptide scaffolds. Grafting MC-12 into the SFTI-1 cyclic scaffold enhances its bioactivity in a mouse model of acute colitis and stability in human serum, indicating that this approach might be useful for the design of novel drug leads for the treatment of colitis and other inflammatory diseases.

Cyc-MC12 had a significant effect on weight loss, macroscopic score, and colon length in the TNBS mouse colitis model. By contrast, MC-12 did not improve weight loss or the macroscopic score relative to the TNBS-treated mice. A significant difference was observed between the colon length of MC-12-treated mice and TNBS-only-treated mice but not to the same extent as cyc-MC12. Both MC-12 and cyc-MC12 were injected into mice at a dose of $3 \mathrm{mg} / \mathrm{kg}$, but MC-12 has a significantly lower molecular weight than cyc-MC12 (MC-12: 445.47 $\mathrm{g} / \mathrm{mol}$; cyc-MC12 $1570.81 \mathrm{~g} / \mathrm{mol}$ ) corresponding to a higher molar concentration. The original study on MC-12 in colitis required a dose of $25 \mathrm{mg} / \mathrm{kg}$ to elicit an influence on macroscopic score and colon length in a TNBS mouse model (18); no effect on weight loss was observed. This improvement in bioactivity of MC-12 in vivo upon grafting in the cyclic scaffold is likely to be related to the significant improvement in stability in serum of cyc-MC12 compared with MC-12.

The three-dimensional structure of cyc-MC12, determined by NMR spectroscopy, is well defined, including the grafted Gln-Ala-Trp sequence. The residues corresponding to MC-12 form a helical structure in the annexin A1 protein (27) as shown in Fig. 1 but do not form a helix when grafted into the cyclic scaffold. This is not surprising given the tightly folded structure of SFTI-1 resulting from the cyclic backbone and cross-bracing of the disulfide bond. Given the well defined nature of the cyclic peptide, it is unlikely it can change conformation significantly to interact with a binding partner. However, based on the activity observed in the TNBS mouse model, the turn structure induced by grafting into the cyclic backbone appears to be sufficient to allow binding to the biological target. The biological target for cyc-MC12 is unknown, but the original studies on the MC-12 tripeptide suggested that NF- $\kappa \mathrm{B}$, a central mediator of gastrointestinal inflammation in IBD, might be involved (18, 28). Further study on these peptides is required to elucidate the mechanism of action. 




Figure 4. Structural analysis of cyc-MC12. The three-dimensional structure of the 20 lowest energy structures of cyc-MC12 is shown on the left of the diagram, highlighting the well defined nature of the peptide, including the grafted region. The PDB ID code is 5VAV, and the BMRB (Biological Magnetic Resonance Data Bank) code is 30274 . The lowest energy structure for cyc-MC12 is shown in the middle of the diagram with the side chains of the grafted residues included. The structure of SFTI- 1 is shown on the right of the diagram with the side chains of the residues replaced with MC-12 shown. The figure was made using MOLMOL (37).

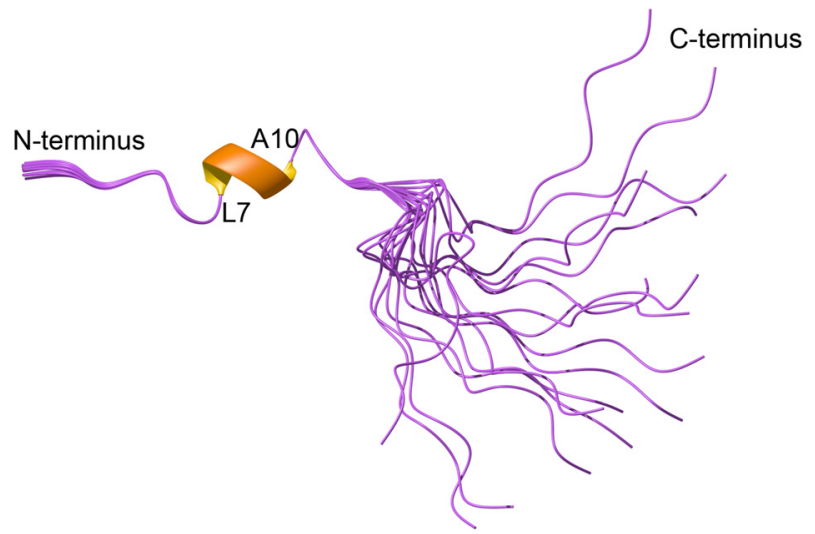

Figure 5. Three-dimensional structure of Ac2-26. The three-dimensional structure of the 20 lowest energy structures was determined using NMR derived constraints. The helical region is shown with a thickened ribbon. The PDB ID code is 5VFW and the BMRB code is 30281 . The figure was made using MOLMOL (37).

Although the binding partner of MC-12 has not been conclusively shown, our current study provides significant insight into the structure function relationships involved in the anti-inflammatory activity. The importance of the cyclic backbone in SFTI-1 grafting has been highlighted in the current study. Previous studies have shown that the disulfide bond and network of hydrogen bonds are sufficient to maintain the native fold (29). However, in the current study we have shown that although the native fold is maintained in the absence of the cyclic backbone in lin-MC12(n), the bioactivity is not maintained. Cyc-MC12 is structurally more stable than lin-MC12(n) based on the slower amide exchange rates and is more biologically stable in human serum than lin-MC12(n). This lack of stability of lin-MC12(n) might account for the lack of bioactivity in vivo. Interestingly, when the MC-12 peptide is grafted into the SFTI-1 binding loop, without backbone cyclization in lin-MC12(p), the chemical shift analysis indicates that the overall fold of the native peptide is not maintained. This peptide has a large extended $\mathrm{N}$-terminal tail that presumably prevents the native structure from forming.

Further insight into the structure function relationships of MC-12 have come from analysis of Ac2-26. Ac2-26 is effective in a range of anti-inflammatory assays including models of ocular inflammation and asthma at doses of $1 \mathrm{mg} / \mathrm{kg}(30,31)$, but the structure has not been reported. We show here that the $\mathrm{N}$-terminal region, including the $\mathrm{MC}-12$ sequence (residues
$9-11$ ), is well defined in contrast to the $C$-terminal region of the peptide (Fig. 5). Residues 9-11 in Ac2-26 overlay well with the corresponding residues in the full-length annexin A1 protein as shown in Fig. 8, but the adjacent residues in Ac2-26 do not overlay with the full-length protein. The conservation of the native structure of $\mathrm{MC}-12$ in the Ac2-26 peptide suggests there will be less entropic losses upon binding to the biological target and consequently why this tripeptide has previously been shown to be so important in anti-inflammatory activity (13). In the current study we have shown that Ac2-26 is not as effective as cyc-MC12 in the colitis model, and this lower efficacy is likely to be related to the lower biological stability; Ac2-26 is degraded rapidly in human serum.

In summary, the potency and stability of the MC-12 tripeptide was improved by incorporation into the SFTI-1 scaffold. The cyclic backbone is important for structural and biological stability and consequently biological activity. Overall, our results indicate that SFTI-1 is a promising scaffold for the design of novel lead molecules for IBD and other diseases that result from a dysregulated immune system.

\section{Experimental procedures}

\section{Peptide synthesis and purification}

Peptides were synthesized using solid-phase peptide synthesis on a Protein Technologies PS3 synthesizer using Fmoc chemistry on a $0.1-\mathrm{mmol}$ scale. Linear peptides were synthesized on 2-chlorotrityl chloride resin. Amino acids (2 eq.) were activated in 0.5 м 2-(1H-benzotriazol-1-yl)-1,1,3,3-tetramethyluronium hexafluorophosphate with 10 eq. of dichloromethane. The first amino acid was coupled manually to the resin (Arg was double-coupled). Peptides were cleaved from the resin using trifluoroacetic acid (TFA)/water/triisopropylsilane (95: 2.5:2.5) for $2-3 \mathrm{~h}$, precipitated with diethyl ether, dissolved in $50 \%$ acetonitrile, $0.05 \%$ TFA and subsequently lyophilized. The resulting crude peptides were purified with RP-HPLC on a C-18 preparative column (Phenomenex Jupiter $250 \times 21.2 \mathrm{~mm}$ ) using a $1 \%$ gradient of solvent B (solvent A: 0.05\% TFA; solvent B: $90 \%$ acetonitrile, $0.05 \%$ TFA). Peptides were oxidized in $0.1 \mathrm{M}$ ammonium bicarbonate, $\mathrm{pH} 8-8.5$, and purified using RP-HPLC, and the mass was analyzed using MALDI mass spectrometry.

The cyclic peptide was synthesized using native chemical ligation (32) on Dawson Dbz AM resin (100-200 mesh). The Dawson resin was deprotected with $20 \%$ piperidine in dimeth- 
(A)

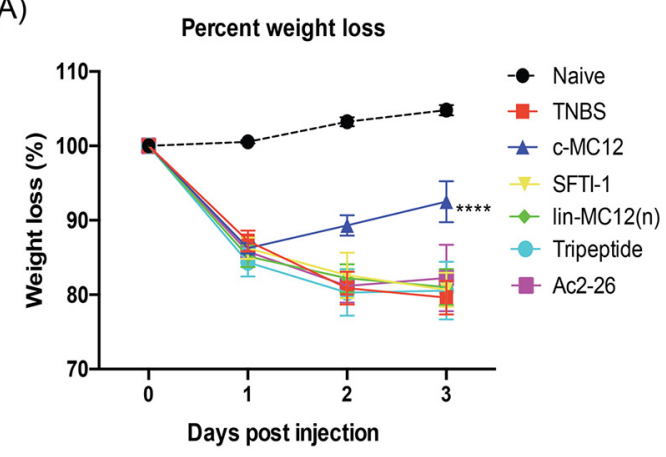

(C)

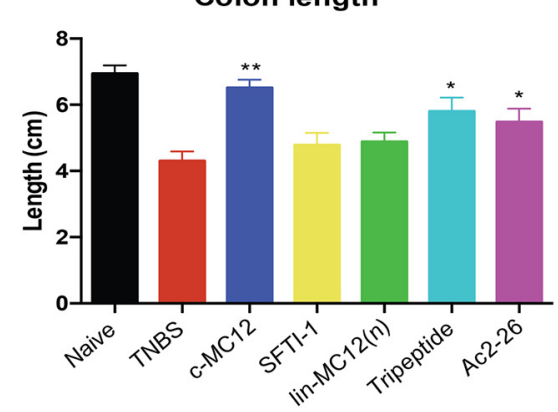

(B)

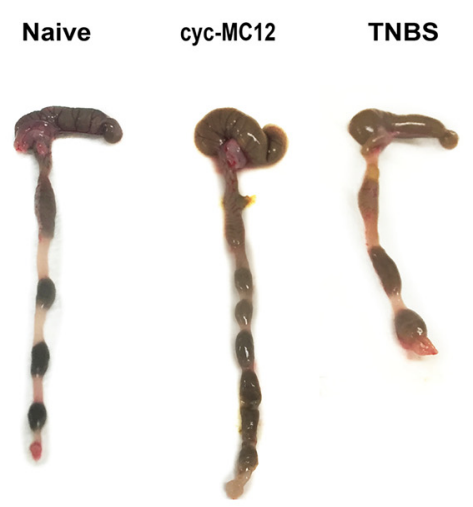

(D)

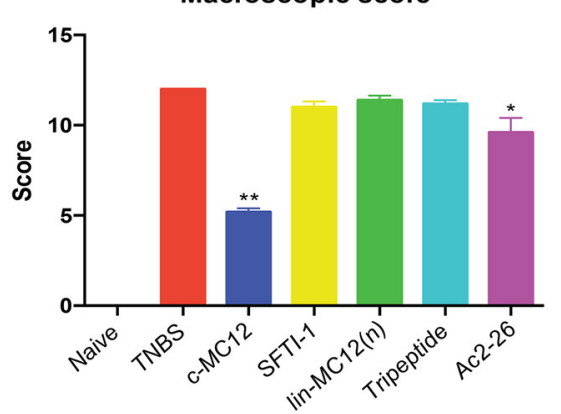

Figure 6. Protective effects of cyc-MC12 against weight loss and clinical symptoms induced by TNBS colitis. Mice were treated with TNBS at day 0 and weighed daily before termination at day 3 . A, percent weight loss $\left.{ }^{* * * *}, p<0.0001\right)$. B, representative photomicrographs of colons at day 3 . $C$, colon lengths at day $3(* *, p=0.0079 ; *, p=0.0397)$. $D$, macroscopic score of colon pathology at day $3(* *, p=0.0079 ; *, p=0.0476)$. All peptides were administered at a dosage of $3 \mathrm{mg} / \mathrm{kg}$ corresponding to injection solution with molar concentrations of $\sim 0.18 \mathrm{~mm}$ for the grafted peptides and $0.1 \mathrm{~mm}$ for Ac2-26. Data show the mean \pm S.E. from a representative experiment of 3 , with $n=5$.

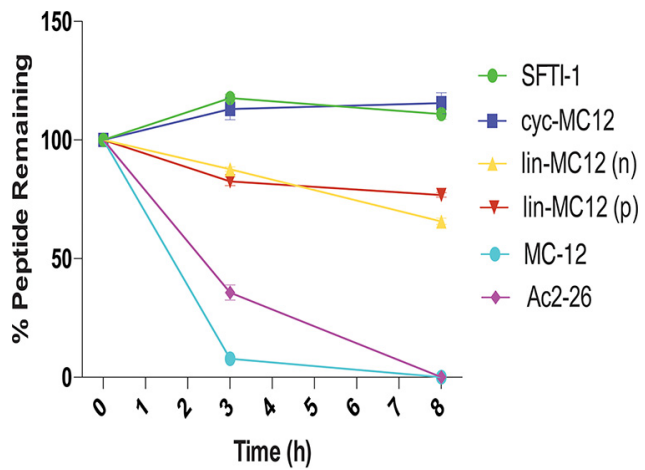

Figure 7. Serum stability of SFTI-1-grafted peptides. The percentage of peptide remaining in the serum stability assay as assessed by RP-HPLC. The grafted peptides and SFTI-1 showed better stability than MC-12 and the longer Ac2-26 peptide. All data are represented as the mean \pm S.D. and were recorded in triplicate.

ylformamide (DMF) $(2 \times 5$ min washes $)$ and then washed with DMF before the addition of the first residue. Before cleavage of the cyclic peptide, acylation of the Dbz linker was achieved by adding 4-nitrophenylchloroformate in dichloromethane (16 eq, room temperature) to the dry resin under $\mathrm{N}_{2}$ for $1 \mathrm{~h}$. The resin was then washed with DMF. $N, N$-Diisopropylethylamine (195 eq.) in DMF was added for $1 \mathrm{~h}$ at room temperature for activation. After activation, the Nbz-peptide was washed with DMF and dried. Cyclization was carried out in $200 \mathrm{~mm} 4$-mercaptophenylacetic acid and $20 \mathrm{~mm}$ tris(2-carboxyethyl) phosphine in $200 \mathrm{~mm}$ phosphate buffer at room temperature for $48 \mathrm{~h}$. The cyclic peptide was purified using RP-HPLC on a C-18



Figure 8. Overlay of Ac2-26 with annexin A1 (PDB ID code 1HM6). Structures were superimposed over residues 9-11 (purple) in Ac2-26 and residues 10-12 (orange) in annexin A1 (these residues correspond to the MC-12 sequence Gln-Ala-Trp). The labeling corresponds to the numbering in cycMC12. The figure was made using MOLMOL (37).

semi preparative column $(250 \times 10 \mathrm{~mm}$ Phenomenex Jupiter C18 column), and mass was analyzed on a 5800 MALDI TOFTOF (SCIEX) mass spectrometer. The cyclic peptide was oxidized using $0.1 \mathrm{M} \mathrm{NH}_{4} \mathrm{HCO}_{4}$. The sample was then purified with RP-HLPC and finally analyzed with NMR.

\section{NMR spectroscopy}

Lyophilized peptides were resuspended to a final concentration of $\sim 0.2 \mathrm{~mm}$ in $90 \% \mathrm{H}_{2} \mathrm{O}: 10 \% \mathrm{D}_{2} \mathrm{O} .2 \mathrm{D}{ }^{1} \mathrm{H},{ }^{1} \mathrm{H}$ TOCSY, ${ }^{1} \mathrm{H},{ }_{1}^{1} \mathrm{H}$ NOESY, ${ }^{1} \mathrm{H},{ }^{1} \mathrm{H}$ DQF-COSY, ${ }^{1} \mathrm{H},{ }^{15} \mathrm{~N}$ HSQC , and ${ }^{13} \mathrm{C},{ }^{1} \mathrm{H}$ HSQC spectra were acquired at $290 \mathrm{~K}$ using a $600 \mathrm{MHz}$ 
AVANCE III NMR spectrometer (Bruker, Karlsruhe, Germany) equipped with a cryogenically cooled probe. All spectra were recorded with an interscan delay of $1 \mathrm{~s}$. NOESY spectra were acquired with mixing times of 200-300 ms, and TOCSY spectra were acquired with isotropic mixing periods of $80 \mathrm{~ms}$. Standard Bruker pulse sequences were used with an excitation sculpting scheme for solvent suppression. Slowly exchanging amide protons were detected by acquiring a series of one-dimensional and TOCSY spectra over a 24-h period after dissolution of the peptides in $\mathrm{D}_{2} \mathrm{O}$. Exchange rates were calculated as previously described for comparison of acyclic and cyclic peptides (33).

The 2D NOESY spectra of cyc-MC12 were automatically assigned and an ensemble of structures was calculated using the program CYANA (34). Torsion-angle restraints from TALOS + were used in the structure calculations. Procheck (35) and Promotif (36) were used to analyze the stereochemical quality of the final structures, which were visualized using MOLMOL (37).

\section{TNBS colitis assay}

All animal experiments were conducted in accordance with the James Cook University Animal Ethics Committee-approved guidelines under the project \#A2003. Groups of five male C57BL/6 mice were used (5 weeks old). Mice were purchased from the Animal Resources Centre (Perth, Australia) and housed in the animal care facility unit at James Cook University under specific pathogen-free conditions (Cairns). Mice were placed inside plastic cages with unlimited access to food and water.

Mice were divided randomly into 8 groups: naïve, TNBS, cyc-MC12, SFTI-1, lin-MC12(n), lin-MC12(p), MC12, and annexin A1 (2-26). Experimental mice received intraperitoneal injections of peptides at a dosage of $3 \mathrm{mg} / \mathrm{kg}$ body weight. The mice were anesthetized before administration of TNBS using mild ketamine/xylazine solution. After anesthesia, each mouse received $100 \mu \mathrm{l}$ of $5 \%(\mathrm{w} / \mathrm{v})$ TNBS solution in $60 \%$ ethanol by intra-colonic instillation using a 20-gauge soft catheter (Terumo), which was inserted into the colon. Following the procedure, the animals were kept vertical for $30 \mathrm{~s}$. Mice were monitored daily for piloerection, survival, body weight, decreased motor activity, rectal bleeding, and stool consistency. Macroscopic pathology score was calculated for each colon after the mice were culled. Briefly, colons were harvested, opened longitudinally, and washed with sterile phosphate buffer saline then placed under a microscope (Olympus SZ61, $0.67-4.5 \mathrm{x})$. The tissues were assessed for changes in macroscopic appearance and scored for pathological changes as follows: adhesion $(0-3)$, bowel wall thickening $(0-3)$, mucosal edema (0-3), ulceration ( $0-3)$, and colon length as described previously (38). All animal experiments were conducted in duplicate to ensure reproducibility of the findings.

\section{Serum stability assay}

The stability of the peptides was tested in human male $A B$ plasma (Sigma) using methods previously described (22). Peptides (final concentration of $200 \mu \mathrm{M}$ ) were incubated in serum or PBS at $37{ }^{\circ} \mathrm{C}$, and $40-\mu \mathrm{l}$ aliquots were taken at $0 \mathrm{~h}, 3 \mathrm{~h}$, and
$8 \mathrm{~h}$. The aliquots of serum were quenched with $40 \mu \mathrm{l}$ of $20 \%$ TFA and incubated for $10 \mathrm{~min}$ at $4{ }^{\circ} \mathrm{C}$ to precipitate serum proteins. PBS received the same treatment as serum. The samples were then centrifuged at $17,000 \times g$ for $10 \mathrm{~min}$, and $90 \mu \mathrm{l}$ of supernatant was analyzed by RP-HPLC at a flow rate of 0.3 $\mathrm{ml} / \mathrm{min}$ using an Phenomenex Jupiter C12 analytical column $(150 \times 2.00 \mathrm{~mm}, 4 \mu \mathrm{m}, 90 \AA)$ using a linear $1 \% \mathrm{~min}^{-1}$ acetonitrile gradient $(0-50 \%$ solvent $\mathrm{B})$. The eluent was observed by dual wavelength UV detector set to 214 and $280 \mathrm{~nm}$.

Author contributions-A. L. and N. L. D. designed the study and, along with C. C. C., wrote the paper. C. C. C., P. S. B., D. W., and L. D. synthesized and purified the peptides. D. W. carried out the mass spectrometry analyses. C. C. C. and N. L. D. determined the structure of the cyclic peptide. C. C. C., S. N., and P. G. carried out the animal experiments. All authors analyzed the results and approved the final version of the manuscript.

\section{References}

1. Ament, M. E. (1975) Inflammatory disease of the colon: ulcerative colitis and Crohns colitis. J. Pediatr. 86, 322-334

2. Strong, S. A. (2001) Surgical management of Crohn's disease. In Surgical treatment: Evidence-based and problem-oriented (Holzheimer R.G., and Mannick J.A., eds), pp. 1-8, Zuckschwerdt, Munich

3. Navarro, S., Ferreira, I., and Loukas, A. (2013) The hookworm pharmacopoeia for inflammatory diseases. Int. J. Parasitol. 43, 225-231

4. Molnár, T., Farkas, K., Szepes, Z., Nagy, F., Szcs, M., Nyári, T., Bálint, A., and Wittmann, T. (2014) Long-term outcome of cyclosporin rescue therapy in acute, steroid-refractory severe ulcerative colitis. United European Gastroenterol. J. 2, 108-112

5. Wangchuk, P., Navarro, S., Shepherd, C., Keller, P. A., Pyne, S. G., and Loukas, A. (2015) Diterpenoid alkaloids of Aconitum laciniatum and mitigation of inflammation by 14-O-acetylneoline in a murine model of ulcerative colitis. Sci. Rep. 5, 12845

6. Leoni, G., Neumann, P. A., Kamaly, N., Quiros, M., Nishio, H., Jones, H. R., Sumagin, R., Hilgarth, R. S., Alam, A., Fredman, G., Argyris, I., Rijcken, E., Kusters, D., Reutelingsperger, C., Perretti, M., Parkos, C. A., Farokhzad, O. C., Neish, A. S., and Nusrat, A. (2015) Annexin A1-containing extracellular vesicles and polymeric nanoparticles promote epithelial wound repair. J. Clin. Invest. 125, 1215-1227

7. Dalmasso, G., Charrier-Hisamuddin, L., Nguyen, H. T., Yan, Y., Sitaraman, S., and Merlin, D. (2008) PepT1-mediated tripeptide KPV uptake reduces intestinal inflammation. Gastroenterology 134, 166-178

8. Akgül, S., Erbil, Y., Giris, M., Alis, H., Yanik, B. T., Olgaç, V., and Toker, G. A. (2006) The effect of octreotide on pancreatic damage in TNBSinduced colitis. Surg. Innov. 13, 102-108

9. Wada, S., Sato, K., Ohta, R., Wada, E., Bou, Y., Fujiwara, M., Kiyono, T., Park, E. Y., Aoi, W., Takagi, T., Naito, Y., and Yoshikawa, T. (2013) Ingestion of low dose pyroglutamyl leucine improves dextran sulfate sodiuminduced colitis and intestinal microbiota in mice. J. Agric. Food Chem. 61, $8807-8813$

10. Bettenworth, D., Buyse, M., Böhm, M., Mennigen, R., Czorniak, I., Kannengiesser, K., Brzoska, T., Luger, T. A., Kucharzik, T., Domschke, W., Maaser, C., and Lügering, A. (2011) The tripeptide KdPT protects from intestinal inflammation and maintains intestinal barrier function. Am. J. Pathol. 179, 1230-1242

11. Kovacs-Nolan, J., Zhang, H., Ibuki, M., Nakamori, T., Yoshiura, K., Turner, P. V., Matsui, T., and Mine, Y. (2012) The PepT1-transportable soy tripeptide VPY reduces intestinal inflammation. Biochim. Biophys. Acta 1820, 1753-1763

12. Flower, R. J. (1988) 11th gaddum memorial lecture: lipocortin and the mechanism of action of the glucocorticoids. Br. J. Pharmacol. 94, 987-1015 


\section{Novel anti-inflammatory peptide}

13. Zhang, Z., Huang, L., Zhao, W., and Rigas, B. (2010) Annexin 1 induced by anti-inflammatory drugs binds to NF- $\kappa \mathrm{B}$ and inhibits its activation: anticancer effects in vitro and in vivo. Cancer Res. 70, 2379-2388

14. de Paula-Silva, M., Barrios, B. E., Macció-Maretto, L., Sena, A. A., Farsky, S. H., Correa, S. G., and Oliani, S. M. (2016) Role of the protein annexin A1 on the efficacy of anti-TNF treatment in a murine model of acute colitis. Biochem. Pharmacol. 115, 104-113

15. Cirino, G., Cicala, C., Sorrentino, L., Ciliberto, G., Arpaia, G., Perretti, M., and Flower, R. J. (1993) Antiinflammatory actions of an N-terminal peptide from human lipocortin-1. Br. J. Pharmacol. 108, 573-574.

16. Stuqui, B., de Paula-Silva, M., Carlos, C. P., Ullah, A., Arni, R. K., Gil, C. D., and Oliani, S. M. (2015) Ac2-26 mimetic peptide of annexin A1 inhibits local and systemic inflammatory processes induced by Bothrops moojeni venom and the Lys -49 phospholipase $\mathrm{A}_{2}$ in a rat model. PLoS ONE 10, e0130803

17. Perretti, M., and D'Acquisto, F. (2009) Annexin A1 and glucocorticoids as effectors of the resolution of inflammation. Nat. Rev. Immunol. 9, 62-70

18. Ouyang, N., Zhu, C., Zhou, D., Nie, T., Go, M. F., Richards, R. J., and Rigas, B. (2012) MC-12, an annexin A1-based peptide, is effective in the treatment of experimental colitis. PLOS ONE 7, e41585

19. Luckett, S., Garcia, R. S., Barker, J. J., Konarev, A. V., Shewry, P. R., Clarke, A. R., and Brady, R. L. (1999) High-resolution structure of a potent, cyclic proteinase inhibitor from sunflower seeds. J. Mol. Biol. 290, 525-533

20. Daly, N. L., Chen, Y. K., Foley, F. M., Bansal, P. S., Bharathi, R., Clark, R. J., Sommerhoff, C. P., and Craik, D. J. (2006) The absolute structural requirement for a proline in the $\mathrm{P} 3^{\prime}$-position of Bowman-Birk protease inhibitors is surmounted in the minimized SFTI-1 scaffold. J. Biol. Chem. 281, $23668-23675$

21. Swedberg, J. E., Nigon, L. V., Reid, J. C., de Veer, S. J., Walpole, C. M., Stephens, C. R., Walsh, T. P., Takayama, T. K., Hooper, J. D., Clements, J. A., Buckle, A. M., and Harris, J. M. (2009) Substrate-guided design of a potent and selective kallikrein-related peptidase inhibitor for kallikrein 4. Chem. Biol. 16, 633-643

22. Chan, L. Y., Gunasekera, S., Henriques, S. T., Worth, N. F., Le, S. J., Clark, R. J., Campbell, J. H., Craik, D. J., and Daly, N. L. (2011) Engineering pro-angiogenic peptides using stable, disulfide-rich cyclic scaffolds. Blood $118,6709-6717$

23. Jendrny, C., and Beck-Sickinger, A. G. (2016) Inhibition of kallikrein-related peptidases 7 and 5 by grafting serpin reactive-center loop sequences onto sunflower trypsin inhibitor-1 (SFTI-1). Chembiochem 17, 719-726

24. Chan, L. Y., Craik, D. J., and Daly, N. L. (2016) Dual-targeting anti-angiogenic cyclic peptides as potential drug leads for cancer therapy. Sci. Rep. 6, 13

25. Quimbar, P., Malik, U., Sommerhoff, C. P., Kaas, Q., Chan, L. Y., Huang, Y. H., Grundhuber, M., Dunse, K., Craik, D. J., Anderson, M. A., and Daly, N. L. (2013) High-affinity cyclic peptide matriptase inhibitors. J. Biol. Chem. 288, 13885-13896
26. Wishart, D. S., Bigam, C. G., Holm, A., Hodges, R. S., and Sykes, B. D. (1995) ${ }^{1} \mathrm{H},{ }^{13} \mathrm{C}$ and ${ }^{15} \mathrm{~N}$ random coil NMR chemical shifts of the common amino acids. I. Investigations of nearest-neighbor effects. J. Biomol. NMR 5, 67-81

27. Perretti, M., D'Acquisto, F., and Flower, R. J. (2013) Annexin-A1 peptide Ac2-26 A2. In Handbook of Biologically Active Peptides (Second Edition) (Kastin, A. J., ed) pp. 631-639, Academic Press, Boston

28. Tambuwala, M. M. (2016) Natural nuclear factor $\kappa \beta$ inhibitors: safe therapeutic options for inflammatory bowel disease. Inflamm. Bowel Dis. 22, $719-723$

29. Korsinczky, M. L., Schirra, H. J., and Craik, D. J. (2004) Sunflower trypsin inhibitor-1. Curr. Protein Pept. Sci. 5, 351-364

30. Girol, A. P., Mimura, K. K., Drewes, C. C., Bolonheis, S. M., Solito, E., Farsky, S. H., Gil, C. D., and Oliani, S. M. (2013) Anti-inflammatory mechanisms of the annexin A1 protein and its mimetic peptide Ac2-26 in models of ocular inflammation in vivo and in vitro. J. Immunol. 190, $5689-5701$

31. Wang, L. M., Li, W. H., Xu, Y. C., Wei, Q., Zhao, H., and Jiang, X. F. (2011) Annexin 1-derived peptide Ac2-26 inhibits eosinophil recruitment in vivo via decreasing prostaglandin D-2. Int. Arch. Allergy Immunol. 154, $137-148$

32. Gunasekera, S., Aboye, T. L., Madian, W. A., El-Seedi, H. R., and Göransson, U. (2013) Making ends meet: microwave-accelerated synthesis of cyclic and disulfide rich proteins via in situ thioesterification and native chemical ligation. Int. J. Pept. Res. Ther. 19, 43-54.

33. Daly, N. L., and Craik, D. J. (2000) Acyclic permutants of naturally occurring cyclic proteins: characterization of cystine knot and $\beta$-sheet formation in the macrocyclic polypeptide kalata B1. J. Biol. Chem. 275, $19068-19075$

34. Güntert, P. (2004) Automated NMR structure calculation with CYANA. In Protein NMR Techniques (Downing, A. K., ed.) pp. 353-378, Humana Press, Totowa, NJ

35. Laskowski, R. A., Macarthur, M. W., Moss, D. S., and Thornton, J. M. (1993) Procheck: a program to check the stereochemical quality of protein structures. J. Appl. Cryst. 26, 283-291

36. Hutchinson, E. G., and Thornton, J. M. (1996) PROMOTIF: a program to identify and analyze structural motifs in proteins. Protein Sci. 5, 212-220

37. Koradi, R., Billeter, M., and Wüthrich, K. (1996) MOLMOL: a program for display and analysis of macromolecular structures. J. Mol. Graph. 14, $51-55$

38. Ferreira, I., Smyth, D., Gaze, S., Aziz, A., Giacomin, P., Ruyssers, N., Artis, D., Laha, T., Navarro, S., Loukas, A., and McSorley, H. J. (2013) Hookworm excretory/secretory products induce interleukin-4 (IL-4)(+) IL$10(+) \mathrm{CD} 4(+) \mathrm{T}$ cell responses and suppress pathology in a mouse model of colitis. Infect. Immun. 81, 2104-2111 